\title{
Theoretical Consideration of Wetting in Cassie-Baxter State on Multi-Pillar and Multi-Hole Surfaces: Thermodynamics and Laplace Pressure
}

\author{
Hiroyuki Mayama ${ }^{1 *}$, Tomoki Nishino², Atsushi Sekiguchi ${ }^{2,3}$, \\ Ryo Nishimura ${ }^{4}$, Kingo Uchida ${ }^{4}$, Satoshi Yokojima ${ }^{5,6}$, \\ Shinichiro Nakamura ${ }^{6}$, and Yoshinume Nonomura ${ }^{7}$ \\ ${ }^{1}$ Department of Chemistry, Asahikawa Medical University, \\ 2-1-1-1 Midorigaoka-higashi, Asahikawa 078-8510, Japan \\ ${ }^{2}$ Department of Mechanical Engineering, Ritumeikan University, \\ 1-1-1 Noji-higashi, Kusatsu, Shiga 525-8577, Japan \\ ${ }^{3}$ Litho Tech Japan Corporation, 2-6-6 Namiki, Kawaguchi, Saitama 332-0034, Japan \\ ${ }^{4}$ Department of Materials Chemistry, Faculty of Science and Technology, Ryukoku University, \\ Seta, Otsu 520-2194, Japan \\ ${ }^{5}$ Schoo of Pharmacy, Tokyo University of Pharmacy and Life Sciences, \\ 1432-1 Horinouchi, Hachioji, Tokyo 192-0392, Japan \\ ${ }^{6}$ RIKEN Science, Technology and Innovation Hub, Nakamura Laboratory, \\ 2-1 Hirosawa, Wako, Saitama 351-0198, Japan \\ ${ }^{7}$ Department of Biochemical Engineering, Graduate School of Science and Engineering, \\ Yamagata University, 4-3-16 Jonan, Yonezawa, Yamagata 992-8510, Japan \\ *mayama@asahikawa-med.ac.jp
}

\begin{abstract}
Wetting in Cassie-Baxter state on multi-pillar and multi-hole surfaces is discussed in terms of Gibbs free energy. To discuss thermodynamic stability, free-energy differences before and after the wetting are formulated. However, no difference between the Cassie-Baxter states on multipillar and multi-hole surfaces is found. Furthermore, Laplace pressure generated by pillars or by a hole is discussed to find the conditions to prevent the penetration of water into the space between the pillars and the hole. As a result, it is predicted the condition in which multi-hole surface easily generates higher Laplace pressure than multi-pillar surface and easy to prevent the penetration. In other words, it is found that the multi-hole surface is a promising way to generate the Cassie-Baxter state.
\end{abstract}

Keywords: Multi-pillar surface, Multi-hole surface, Gibbs free energy, Laplace pressure

\section{Introduction}

Various wetting phenomena such as bouncing and rolling raindrops on lotus leaves and tears of wine on wine glasses can be seen in our daily lives [1-10]. In principle, wetting phenomena are divided to two aspects [11]. One is static wetting. This is mainly discussed in terms of hydrophobicity, contact angle, wetting of structured surface in Wenzel state and Cassie-Baxter state (CB state). These can be discussed by thermodynamics based on Gibbs free energy. Other is dynamic wetting such as spreading, hydrodynamics of interface, transport phenomena, impacts and bounces of water droplets, etc. Wetting phenomena have been intensively studied from fundamental study to application using multi-pillar surface (MP surface), where MP surface is prepared by micro-fabrication using $\mathrm{Si}$ wafer and it has a surface structure in which fine pillars are periodically arranged [4-10]. Multi-hole surface (MH surface) is also important because it can be prepared by micro-printing and molding and it is also important in micro-fabrication. However, the experiments on wetting phenomena on $\mathrm{MH}$ surface have been rarely reported. In this article, we focus 
(a)

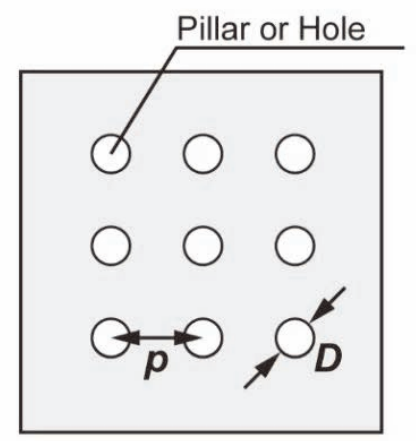

(b)

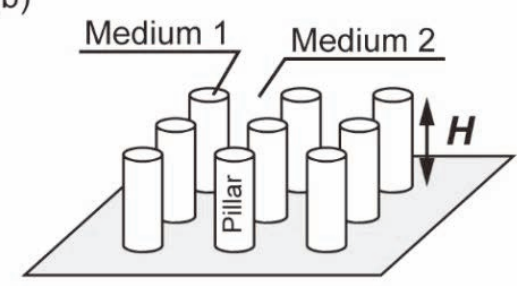

(c)

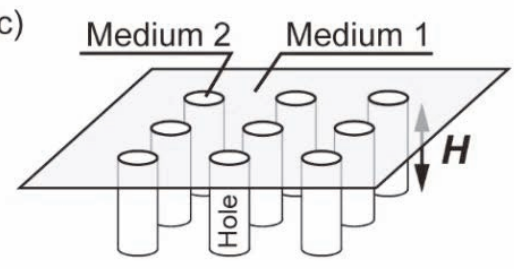

(a)

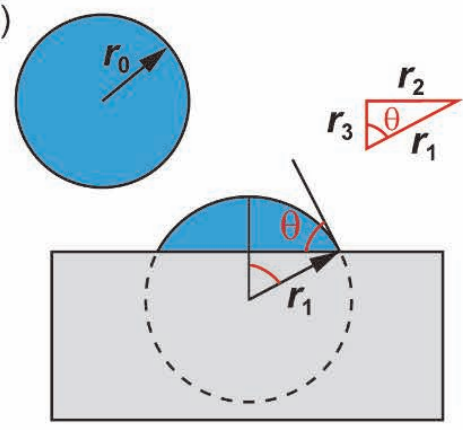

(b)

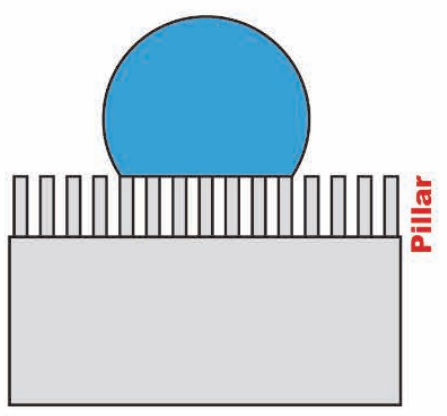

(c)

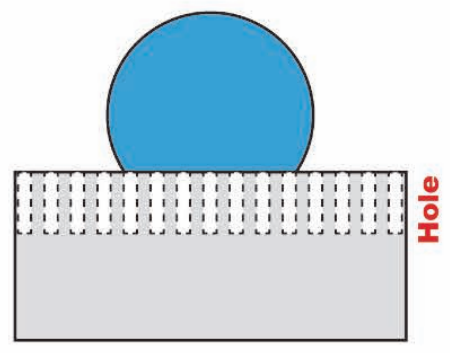

Fig. 2. Schematic representations of wetting on flat surface (a), wetting in the CB state on multi-pillar surface (b) and multi-hole surface (c).

$$
\Delta G=G_{f}-G_{i} .
$$

Here, it should be noted that interfacial areas between solid, air and liquid are included in $G_{i}$ and $G_{f} . \quad G_{i}$ and $G_{f}$ are formulated by the interfacial area and surface tensions.

$$
\begin{gathered}
G_{i}=\gamma_{S} A_{S}+\gamma_{L} A_{L}, \\
G_{f}=\gamma_{S} A_{S}^{\prime}+\gamma_{S L} A_{S L}^{\prime}+\gamma_{L} A_{L}^{\prime},
\end{gathered}
$$

where $A$ is interfacial area, $\gamma$ is surface tension, suffixes of $S, L$ and $S L$ mean the air-solid interface, the air-liquid interface and the solid-liquid interface, respectively. Here, $A$ without and with primes ( $\left.{ }^{\prime}\right)$ means the interfacial areas before and after wetting, respectively. $A_{S}^{\prime}$ in Eq. (3) is related with $A_{S}$ and $A_{S L}^{\prime}$ (wetting area) as described by Eq. (4).

$$
A_{S}^{\prime}=A_{S}-A_{S L}^{\prime}
$$

From Eqs. (2), (3) and (4), $\Delta G$ is obtained as Eq. (5). 


$$
\begin{aligned}
\Delta G & =G_{f}-G_{i} \\
& =\left(\gamma_{S L}-\gamma_{S}\right) A_{S L}^{\prime}+\gamma_{L}\left(A_{L}^{\prime}-A_{L}\right) .(5)
\end{aligned}
$$

It should be noted that surface structure of substrate is reflected in $A_{S L}^{\prime}$.

Based on Eq. (5), we discuss $\Delta G$ on wetting of flat surface, multi-pillar surface and multi-hole surface. In the discussion, we assume the following points: water droplet is small, but, it is significantly larger than the surface structures, water droplets are not deformed by gravity, and the shape of the air-liquid interface relating $A_{L}^{\prime}$ is hemispherical. Figure 2a shows a schematic representation of wetting on substrate. $A_{S L}^{\prime}, A_{L}^{\prime}$ and $A_{L}$ in Eq. (5) are functions of radius of the droplet before wetting $r_{0}$, contact angle $\theta$ as shown below.

\section{2. $\Delta G$ in wetting on flat surface [12]}

First, we discuss $\Delta G$ in wetting on flat surface. The equilibrium contact angle is defined as $\theta_{e q}^{\text {flat }}$. $A_{S L}^{\prime}, A_{L}^{\prime}$ and $A_{L}$ in Eq. (5) are described by Eqs. (6)-(8):

$$
\begin{gathered}
A_{S L}^{\prime}=\pi\left(\frac{4}{2-3 \cos \theta_{e q}^{\text {flat }}+\cos ^{3} \theta_{e q}^{\text {flat }}}\right)^{2 / 3} r_{0}^{2} \sin ^{2} \theta_{e q}^{\text {flat }} \text {. (6) } \\
A_{L}^{\prime}=2 \pi\left(\frac{4}{2-3 \cos \theta_{e q}^{\text {flat }}+\cos ^{3} \theta_{e q}^{\text {flat }}}\right)^{\frac{2}{3}} r_{0}^{2}\left(1-\cos \theta_{e q}^{\text {flat }}\right) \\
A_{L}=4 \pi r_{0}^{2}
\end{gathered}
$$

Using Eqs. (6)-(8), $\Delta G$ is described by Eq. (5) as Eq. (9):

$$
\begin{gathered}
\Delta G=\left(\gamma_{S L}-\gamma_{S}\right) \\
\times \pi\left(\frac{4}{2-3 \cos \theta_{e q}^{\text {flat }}+\cos ^{3} \theta_{e q}^{\text {flat }}}\right)^{\frac{2}{3}} r_{0}^{2} \sin ^{2} \theta_{e q}^{\text {flat }} \\
+\gamma_{L}\left[\begin{array}{c}
2 \pi\left(\frac{4}{2-3 \cos \theta_{e q}^{\text {flat }}+\cos ^{3} \theta_{e q}^{\text {flat }}}\right)^{\frac{2}{3}} \\
\times r_{0}^{2}\left(1-\cos \theta_{e q}^{\text {flat }}\right)
\end{array}\right] \\
-4 \pi \gamma_{L} r_{0}^{2} .
\end{gathered}
$$

Here, we define $\Delta g$, which is $\Delta G$ normalized by $4 \pi r_{0}^{2}$, to understand wetting phenomena only by contact angle.

$$
\Delta g=\frac{\Delta G}{4 \pi r_{0}^{2}}
$$

Equation (10) is very useful to understand thermodynamic stability of wetting state because $\Delta g$ does not depend on volume and surface area of droplet. From the condition of $\partial \Delta g / \partial \theta_{e q}^{\text {flat }}=0$ of Eq. (10), Young's law is obtained.

$$
\cos \theta_{e q}^{\text {flat }}=\frac{\gamma_{S}-\gamma_{S L}}{\gamma_{L}}
$$

3.3. $\Delta G$ of Cassie-Baxter state in wetting on multipillar surface

We assume that wetting state is Cassie-Baxter state on multi-pillar (MP) surface in which water droplets touch the pillar head and do not penetrate into the space between the pillars. To distinguish the contact angle from $\theta_{e q}^{\text {flat }}$, we define the contact angle on the MP surface in the Cassie-Baxter (CB) state as $\theta_{C B}^{M P}$. Originally, the contact angle on the CB state, $\theta_{C B}$, is defined by Eq. (12):

$$
\cos \theta_{C B}=f_{1} \cos \theta_{1}+f_{2} \cos \theta_{2} \text {. }
$$

where the suffixes mean the mediums (1: material and 2: air), $\theta_{1}$ and $\theta_{2}$ are the equilibrium contact angle of material and air, respectively, $f_{1}$ and $f_{2}$ are the area fraction where material (1) and air (2) occupied in the apparent wetting area, respectively, and $f_{1}+f_{2}=1$. Along this line, $\theta_{C B}^{M P}$ can be defined by Eq. (13):

$$
\cos \theta_{C B}^{M P}=f_{1}^{M P} \cos \theta_{1}+f_{2}^{M P} \cos \theta_{2} .
$$

$f_{1}^{M P}$ and $f_{2}^{M P}$ are described by Eqs. (14) and (15):

$$
\begin{gathered}
f_{1}^{M P}=\frac{\pi}{4}\left(\frac{D}{p}\right)^{2} . \\
f_{2}^{M P}=1-\frac{\pi}{4}\left(\frac{D}{p}\right)^{2} .
\end{gathered}
$$

$A_{S L}^{\prime}$ (the apparent wetting area) in Eq. (6) and $A_{L}^{\prime}$ in Eq. (7) are modified as Eqs. (16) and (17):

$$
\begin{aligned}
A_{S L}^{\prime} & =\pi\left(\frac{4}{2-3 \cos \theta_{C B}^{M P}+\cos ^{3} \theta_{C B}^{M P}}\right)^{\frac{2}{3}} r_{0}^{2} \sin ^{2} \theta_{C B}^{M P} \\
& =\left(f_{1}^{M P}+f_{2}^{M P}\right) A_{S L}^{\prime} . \\
A_{L}^{\prime}= & 2 \pi\left(\frac{4}{2-3 \cos \theta_{C B}^{M P}+\cos ^{3} \theta_{C B}^{M P}}\right)^{\frac{2}{3}} r_{0}^{2}\left(1-\cos \theta_{C B}^{M P}\right) .(17)
\end{aligned}
$$

Next, we try to describe the free-energy difference $\Delta G$. From Eqs. (5), (11) and (16), $\Delta G$ is modified as Eq. (18):

$$
\begin{gathered}
\Delta G_{C B}^{M P}=\gamma_{L}\left[1-f_{1}^{M P}\left(1+\cos \theta_{e q}^{\text {flat }}\right)\right] A_{S L}^{\prime} \\
+\gamma_{L}\left(A_{L}^{\prime}-A_{L}\right) .
\end{gathered}
$$

The normalized $\Delta G_{C B}^{M P}, \Delta g_{C B}^{M P}$, is obtained as Eq. (19):

$$
\Delta g_{C B}^{M P}=\frac{\Delta G_{C B}^{M P}}{4 \pi r_{0}^{2}} .
$$

Eq. (19) is useful to understand thermodynamic 


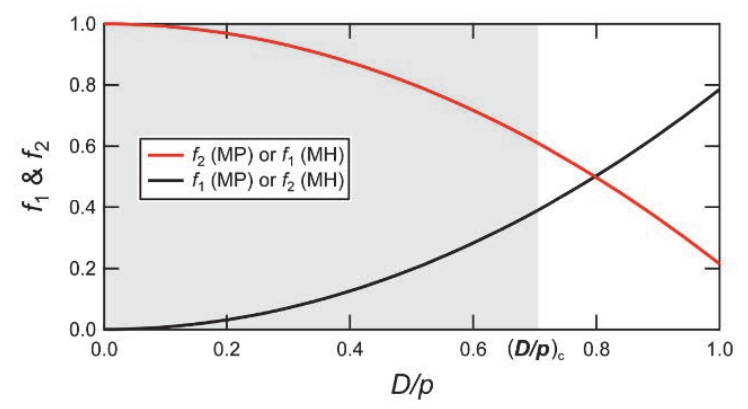

Fig. 3. Dependences of $f_{1}$ and $f_{2}$ on $D / p$ in Eqs. (14), (15), (20) and (21). Here, the suffixes 1 and 2 mean material and air, respectively. The geometries are shown in Figs. 1b and 1c. The shaded area is the condition that Laplace pressure of MH surface is larger than that of MP surface. See, Eq. (44). $(D / p)_{c}=$ $\alpha_{c}=0.707$.

stability of wetting state because $\Delta g_{C B}^{M P}$ is independent on the volume and surface area of droplets.

\section{4. $\Delta G$ of Cassie-Baxter state in wetting on multi-} hole surface

The multi-hole surface and the multi-pillar surface are the relationship between front and back. Therefore, the area fractions in the apparent contact area on multi-hole surface, $f_{1}^{M H}$ and $f_{2}^{M H}$, are obtained from Eqs. (14) and (15) as

$$
\begin{gathered}
f_{1}^{M H}=1-\frac{\pi}{4}\left(\frac{D}{p}\right)^{2} . \\
f_{2}^{M H}=\frac{\pi}{4}\left(\frac{D}{p}\right)^{2} .
\end{gathered}
$$

$A_{S L}^{\prime}$ (the apparent wetting area) in Eq. (6) and $A_{L}^{\prime}$ in Eq. (7) are modified as Eqs. (22) and (23):

$$
\begin{aligned}
A_{S L}^{\prime} & =\pi\left(\frac{4}{2-3 \cos \theta_{C B}^{M H}+\cos ^{3} \theta_{C B}^{M H}}\right)^{\frac{2}{3}} r_{0}^{2} \sin ^{2} \theta_{C B}^{M H} \\
& =\left(f_{1}^{M H}+f_{2}^{M H}\right) A_{S L}^{\prime} . \\
A_{L}^{\prime} & =2 \pi\left(\frac{4}{2-3 \cos \theta_{C B}^{M H}+\cos ^{3} \theta_{C B}^{M H}}\right)^{\frac{2}{3}} r_{0}^{2}\left(1-\cos \theta_{C B}^{M H}\right) .
\end{aligned}
$$

Free-energy difference $\Delta G_{C B}^{M H}$ and its normalized one $\Delta g_{C B}^{M H}$ are

$$
\begin{gathered}
\Delta G_{C B}^{M H}=\gamma_{L}\left[1-f_{1}^{M H}\left(1+\cos \theta_{e q}^{f l a t}\right)\right] A_{S L}^{\prime} \\
+\gamma_{L}\left(A_{L}^{\prime}-A_{L}\right) . \\
\Delta g_{C B}^{M H}=\frac{\Delta G_{C B}^{M H}}{4 \pi r_{0}^{2}} .
\end{gathered}
$$
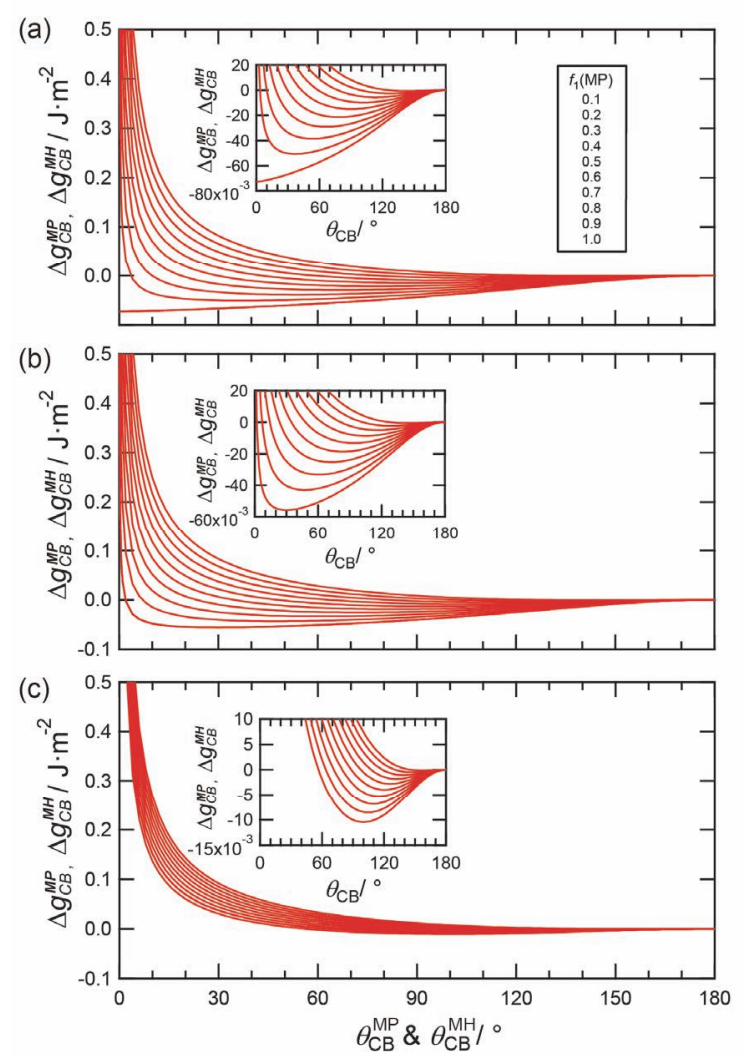

Fig. 4. Dependences of normalized free-energy difference in Cassie-Baxter states on multi-pillar surfaces and multi-hole surfaces on contact angle $\theta_{C B}$ by Eqs. (19) and (25). The conditions are $\gamma_{L}=$ $72.8 \mathrm{mN} / \mathrm{m}$ in (a)-(c), and $\theta_{\text {eq }}^{\text {flat }}=0^{\circ}, 30^{\circ}$ and $100^{\circ}$ in (a), (b) and (c), respectively. The profiles are plotted every $f_{1}^{M P}=f_{1}^{M H}=0.1$. Here, it should be noted that the profiles at $f_{1}^{M P}=f_{2}^{M H}=1.0$ are equal to the profiles on flat surfaces. The insets enlarge the vicinities of free-energy minimum. The order of the profiles from top to bottom obey that of the explanatory notes. The medium 1 is material and the medium 2 is air.

3.5. Computational results of the normalized freeenergy differences of wetting on multi-pillar and multi-hole surfaces

Figure 3 shows the relationship between $f_{1}^{M P}$, $f_{2}^{M P}, f_{1}^{M H}, f_{2}^{M H}$ and $D / p$. The magnitude relationship between $f_{1}^{i}$ and $f_{2}^{i}$ is exchanged at $D / p=0.798$, where $i$ means MP and MH. On the other hand, Figure 4 shows the profiles of the normalized free-energy differences of the CB states on the MP and MH surfaces as function of the contact angles. In the case of the MP surface, the free-energy minimum shifts to higher contact angle with the decrease in $f_{1}$ because the space between the pillars is apart. For example, even if the material is superhydrophilic $\left(\theta_{e q}=0^{\circ}\right)$, the stable 
contact angle increases to $90^{\circ}$ at $f_{1}=0.4$. If the material is hydrophilic $\left(\theta_{e q}=30^{\circ}\right)$, the stable contact angle increases to $90^{\circ}$ at $f_{1}=0.5$. If the material is hydrophobic $\left(\theta_{e q}=100^{\circ}\right)$, the stable contact angle increases to $150^{\circ}$ at $f_{1}=0.1$. On the other hand, no differences between the MP surface and the MH surfaces are found in terms of the normalized free-energy difference and freeenergy difference. The main reason is that the morphology of these surfaces, MP or MH, is hidden behind $f_{1}$ and $f_{2}$.

\section{Laplace pressure of multi-pillar and multi-hole surfaces}

Among dynamic wetting phenomena, Laplace pressure is generated by surface structures and it is important to understand the bouncing raindrops on lotus leaves. In the following discussion, we try to derive the equations of Laplace pressures generated by MP and MH surfaces. Then, these are compared.

\subsection{Laplace pressure generated by MP surface [4]}

First, we discuss how Laplace pressure $P_{L}$ is generated by multi-pillar surface structure. To discuss $P_{L}$, it is important to consider the curvature radius of dented air-liquid interface between the surface structures $R$, dipping angle $\varphi$ and its dipping depth $d$ as shown Fig. 5a. Dipping is generated by pinning effect for contact line by the edges of pillars, i.e., physical defects. In the geometry in Fig. 5a, $d$ is related to $R$ and $\varphi$ as Eq. (26):

$$
d=R(1-\cos \varphi) \sim \frac{\varphi^{2}}{2} R .
$$

Here, $\cos \varphi \sim 1-\varphi^{2} / 2$ under the conditions of $\varphi<1$ and the unit of $\varphi$ is radian.

On the other hand, the relationship between $R$, $\varphi$ and characteristic sizes of multi-pillar surface (pillar diameter $D$, pillar height $H$, pitch $p$ ) is formulated as Eq. (27):

$$
R \sin \varphi \sim R \varphi=\frac{\sqrt{2} p-D}{2},
$$

here, $\sin \varphi \sim \varphi$ under the conditions of $\varphi \sim 0$. If $\varphi$ reaches the critical dipping angle $\varphi_{c}$, the airliquid interface advances because the edge is a part of the flat surface of the top of pillar and also a part of the flat wall of pillar. Considering the virtual surface extended along the wall of pillar as shown in Fig. 5a, $\varphi_{c}$ is directly related to the equilibrium contact angle on flat surface $\theta_{e q}$ as follows:

$$
\varphi_{c}=\theta_{e q}-\frac{\pi}{2}
$$

(a)

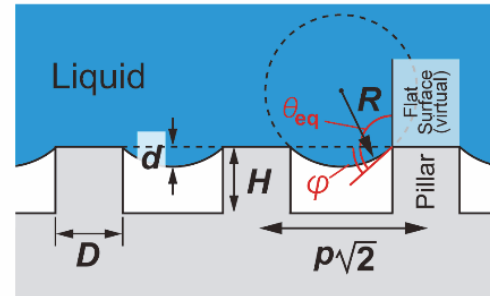

(b)

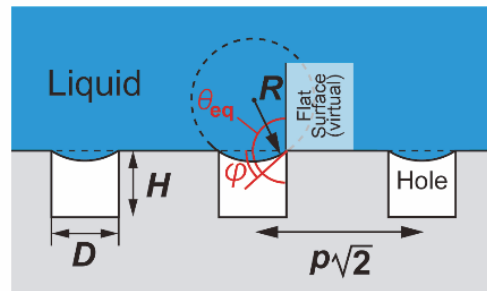

Fig. 5. Schematic representations of cross sections of the air-liquid interface on MP surface with square lattice arrangement of pillars (a) and MH surface (b).

Next, we discuss $P_{L}$ based on Eqs. (26)-(28). In principle, the equation of Laplace pressure is described by Eq. (29):

$$
P_{L}=\frac{2 \gamma_{L}}{R} .
$$

By substitution of $R$ by Eq. (27), (29) is modified as Eq. (30):

$$
P_{L}=\frac{\gamma_{L} \varphi^{2}}{d} .
$$

The dependence, which $P_{L}$ is proportional to squared dipping angle $\varphi_{0}^{2}$, is important to discuss $P_{L}$ on actual multi-pillar surface with tilting pillars later.

From Eqs. (26) and (27), $\varphi^{2}$ is described as Eq. (31):

$$
\varphi^{2}=\frac{(\sqrt{2} p-D)^{2}}{4 R^{2}}=\frac{2 d}{R} .
$$

From Eq. (31),

$$
\frac{1}{d}=\frac{8 R}{(\sqrt{2} p-D)^{2}} .
$$

Using Eqs. (30), (31) and (32), Laplace pressure of multi-pillar surface $P_{L}^{M P}$ becomes Eq. (33):

$$
P_{L}^{M P}=\frac{16 \gamma_{L} d}{(\sqrt{2} p-D)^{2}} .
$$

Under the conditions of $p>d, d$ reaches $H$ and then Eq. (33) is changed as Eq. (34):

$$
P_{L}^{M P}=\frac{16 \gamma_{L} H}{(\sqrt{2} p-D)^{2}} .
$$


Introducing the ratio parameters $\alpha=D / p$ and $x=H / D$, Eq. (34) becomes Eq. (35):

$$
P_{L}^{M P}=\frac{16 \gamma \alpha x}{(\sqrt{2}-\alpha)^{2} p} .
$$

where $0 \leq \alpha \leq 1$.

\subsection{Laplace pressure generated by MH surface}

Next, we discuss Laplace pressure generated by multi-hole surfaces. In Figure $5 \mathrm{~b}, d$ is related to $R$ and $\varphi$ as Eq. (36), which is the same to Eq. (26):

$$
d \sim \frac{\varphi^{2}}{2} R .
$$

On the other hand, the relationship between $R$, $\varphi$ and hole diameter $D$ is formulated under the conditions of $\varphi \sim 0$ as Eq. (37):

$$
R \varphi \sim \frac{D}{2} .
$$

This is different from Eq. (27). (38):

From Eqs. (36) and (37), $\varphi^{2}$ is obtained as Eq.

$$
\varphi^{2} \sim \frac{2 d}{R} \sim \frac{D^{2}}{4 R^{2}} .
$$

Eq. (38) is corresponding to Eq. (31).

Eq. (30) is shown again as Eq. (39):

$$
P_{L}=\frac{\gamma_{L} \varphi^{2}}{d} .
$$

From Eq. (38), Eq. (39) is modified as (40) and Laplace pressure of multi-hole surface $P_{L}^{M H}$ is obtained:

$$
P_{L}^{M H}=\frac{8 \gamma_{L} R \varphi^{2}}{D^{2}}=\frac{8 \gamma_{L} R}{D^{2}} \frac{2 d}{R}=\frac{16 \gamma_{L} d}{D^{2}} .
$$

Under the conditions of $D>H, d$ reaches $H$ and then Eq. (40) is changed as Eq. (41):

$$
P_{L}^{M H}=\frac{16 \gamma_{L} H}{D^{2}} .
$$

Using $\alpha=D / p$ and $x=H / D$, Eq. (41) becomes Eq. (42):

$$
P_{L}^{M H}=\frac{16 \gamma_{L} x}{\alpha p} .
$$

\subsection{Comparison between $P_{L}^{M P}$ and $P_{L}^{M H}$}

Next, let us compare $P_{L}^{M P}$ and $P_{L}^{M H}$ in terms of $\alpha$. When we consider the critical condition of $P_{L}^{M P}=P_{L}^{M H}$, Eq. (43) is obtained using Eqs. (35) and (42):

$$
\frac{16 \gamma \alpha x}{(\sqrt{2}-\alpha)^{2} p}=\frac{16 \gamma_{L} x}{\alpha p} .
$$

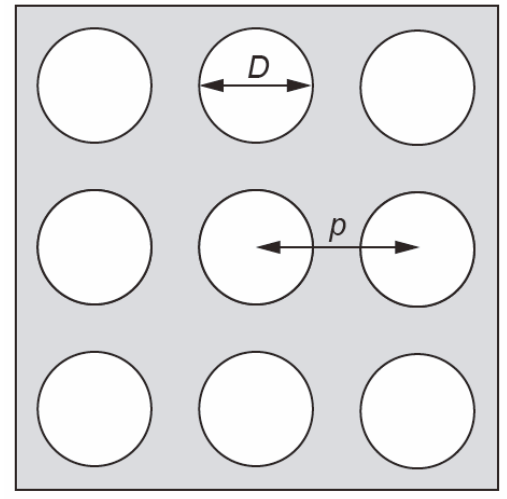

Fig. 6. Schematic representation of the top view of square lattice at $\alpha=D / p=0.707$.

From Eq. (43), the critical condition of $\alpha, \alpha_{c}$, is determined as Eq. (44):

$$
\alpha_{c}=\left(\frac{D}{p}\right)_{c}=\frac{\sqrt{2}}{2}=0.707 .
$$

Figure 6 shows the square pattern of $\alpha_{c}=0.707$. Based on $\alpha_{c}$, it is possible to judge which surface is generates larger Laplace pressure. The condition for $P_{L}^{M P}<P_{L}^{M H}$ is as follows:

$$
\alpha<0.707 \text {. }
$$

Under the conditions of $\alpha<0.707, \mathrm{MH}$ surfaces generate higher Laplace pressure and prevent the penetration of water into the holes. The shaded area in Fig. 3 shows $\alpha<0.707$.

In contrast, the condition for $P_{L}^{M P}>P_{L}^{M H}$, which is the condition to prevent the penetration into the MP surface, is described by Eq. (46):

$$
\alpha>0.707 \text {. }
$$

This means that the space between the pillars on the MP surface is narrow.

For experiments, it is fruitful to calculate the order of $p$ under rough conditions. The values of $p$ and $D$ of the MP surface are estimated to be $2.47 \times 10^{-5}$ and $1.98 \times 10^{-5} \mathrm{~m}$ under the conditions of $x=1$ and $\alpha=0.8$ on the MP surface and $P_{L}^{M P} \sim 10^{5} \mathrm{~Pa}$ (atmospheric pressure). These orders are $\sim 10 \mu \mathrm{m}$. On the other hand, the values of $p$ and $D$ of the MH surface are estimated to be $1.94 \times 10^{-5}$ and $1.16 \times 10^{-5} \mathrm{~m}$ under the conditions of $x=1$ and $\alpha=0.6$ on the MP surface and $P_{L}^{M H} \sim 10^{5} \mathrm{~Pa}$. These orders are also $\sim$ $10 \mu \mathrm{m}$.

Though we estimated Laplace pressures under 
the rough conditions, Laplace pressure would be helpful to design the scales in the MP and $\mathrm{MH}$ surfaces to generate not only the bouncing phenomena, but also to understand CB state.

\section{Conclusion}

We discussed wetting phenomena on MP and MH surfaces based on Gibbs free energy and Laplace pressure. Considering Gibbs free energy of the CB state on MP and MH surfaces, no differences in freeenergy difference are found. In contrast, the difference between MP and MH surfaces in Laplace pressure is found. Laplace pressures are equal at the condition of $D / p=0.707$. If $D / p$ is smaller than 0.707 , then the $\mathrm{MH}$ surfaces generate larger Laplace pressure than that by the MP surfaces. If $D / p$ is larger than 0.707 , the MP surfaces generate larger Laplace pressure. These theoretical findings give us a guiding principle to control dynamic wetting on MP and MH surfaces. Further studies based on this theoretical scenario allow us to design wetting phenomena on surface with suitable structures for application.

\section{Acknowledgement}

This work was supported by a Grant-in-Aid for Scientific Research (C) (JSPS KAKENHI Grant Number JP18K03554).

\section{References}

1. R. Nishimura, K. Hyodo, H. Sawaguchi, Y. Yamamoto, Y. Nonomura, H. Mayama, S. Yokojima, S. Nakamura, and K. Uchida, J. Am. Chem. Soc., 138 (2016) 10299.

2. W. Barthlott and C. Neinhuis, Planta, 202 (1997) 1.

3. M. D. Bartlett, A. B. Croll, D. R. King, B. M. Paret, D. J. Irschick, and A. J. Crosby, $A d v$. Mater., 24 (2019) 1078.

4. Y. C. Jung and B. Bhushan, Langmuir, 25 (2009) 9208.

5. C. C. Chang, C. J. Wu, Y. J. Sheng, and H. K. Tsao, Soft Matter, 11 (2015) 7308.

6. B. Bhushan and E. K. Her, Langmuir, 26 (2019) 8207.

7. A. Tuteja, W. Choi, M. Ma, J. M. Marby, S. A. Mazzella, G. C. Rutledge, G. H. McKinley, and R. E. Cohen, Science, 318 (2007) 1618.

8. A. Tuteja, W. Choi, J. M. Marby, G. H. McKinley, and R. E. Cohen, Proc. Natl. Acad. Sci. USA, 105 (2008) 18200.

9. B. H. Neelesh, A. Patankar, and J. Lee, Langmuir, 19 (2003) 4999.

10. P. S. Forsberg, C. Priest, M. Brinkmann, R. Sedev, and J. Ralston, Langmuir, 26 (2010) 860.

11. P. G. de Gennes, Rev. Mod. Phys., 57 (1985) 827.

12. H. Mayama and Y. Nonomura, Langmuir, 27 (2011) 3550 . 